\title{
Sensitivity Analysis of Incentive Situations and Personal Attributes to Driving Anger Based on MNL Model: A Naturalistic Experimental Study
}

\author{
Ping WAN, Lixin YAN, Shan LU*
}

\begin{abstract}
Driving anger, called "road rage", has gradually become universal phenomenon nowadays, which has been a concern to traffic management authorities. It is necessary to figure out impacting degree of the influencing factors on driving anger for taking the corresponding intervening measures. Forty drivers were enrolled to conduct naturalistic experiments on a busy route in Wuhan, China, where drivers' anger can be induced by various incentive situations including jaywalking, weaving/cutting in line, traffic congestion and red light with extra paid if completing the experiment ahead of reference time. According to behavioral theory and disaggregation theory, the influencing factors including the incentive situations and personal attributes (i.e. gender, age, temperament) were determined for proposing driving anger prediction model based on MNL (multinomial logit). Then, the sensitivity of each influencing factor on driving anger was analyzed by elasticity theory on the basis of the proposed model. The result indicates that age, temperament and illegal behaviors from surrounding people are decisive influencing factors to driving anger sates with different intensity because their average elasticity values for none anger (neutral), low anger, medium anger, high anger are 1.254, 2.713, 2.914, respectively, which are all bigger than 1 . Moreover, the accuracy of the proposed model is $78.30 \%$. The results can provide theoretical support for developing key monitoring or targeted intervention to deal with the decisive influencing factors for traffic management authorities.
\end{abstract}

Keywords: driving anger; incentive factors; MNL; naturalistic experiment; personal attributes

\section{INTRODUCTION}

Driving anger, also called "road rage", is a special driving emotion triggered by incentive situations including adverse traffic environment or discourteous behaviors from surrounding traffic participants [1]. Road rage has become an increasingly common traffic hazard all over the world. A report from American Automobile Association (AAA) in 2009 indicated that 5-7\% of surveyed drivers were perpetrators for "road rage". Moreover, $30 \%$ of the perpetrators are professional drivers including truck and bus drivers [2]. However, about $60 \%$ of drivers in China ever had "road rage" experiences in their daily life [3]. As anger inevitably influences a driver's perception, identification, decision and volition process, the driver will have a degraded driving performance finally [4]. Furthermore, there exists a close relationship between traffic crashes and aggressive driving behaviour which are caused by anger [5]. An angry driver tends to make more mistakes or lapses or violations which result in more involvement in traffic incidents [6]. A statistical report from National Highway Safety Administration of U.S. showed that the ratio of traffic accidents resulted from emotional driving including road rage takes up $9.2 \% \sim 14.8 \%$ of the total [7]. In China, statistics show that road rage resulted in 17.33 million illegal acts, leading to 83100 traffic accidents in $2015,1.22 \%$ higher than that of 2014 [8]. Except for those incentive situations, personal attributes like gender, age, driving experience (years) and temperament also influence driving anger. Therefore, the influencing factors and how the factors impact on driving anger should be excavated for targeted interventions for road rage.

In order to effectively analyze the impact of the influencing factors on driving anger, it is critical to figure out the relationship between the influencing factors and driving anger states with different intensity. Correspondingly, a brief review of related studies regarding the aforementioned aspects is shown in the following paragraphs.

(1) Incentive situations factors
Adverse traffic environment and bad behaviors from surrounding traffic participants (collectively called incentive situations) can make drivers become angry. Deffenbacher et al. conducted a questionnaire survey among 1500 college student drivers to design a driving anger scale (DAS) and found driving anger often occurred in six kinds of incentive situations, namely, rude behavior, hostile gesture, low speed driving, illegal driving, traffic obstruction and police presence [9]. Gonzalez-Iglesias et al. found that Spanish male drivers were prone to become angry when encountering police enforcing the law, while female drivers were inclined to become angry when traffic jam happened [10]. According to DAS statistics, Escanes et al. discovered that female drivers in Argentina were apt to be angry when facing traffic obstruction and hostile gestures from surrounding drivers. Stephens et al. [11] found that when a driver was forced to follow a slowmoving vehicle ahead, their anger level would gradually increase due to time pressure, leading to a more obvious hazardous overtaking intention and more dangerous driving behaviours. Based on a questionnaire survey, Putranto et al. [12] discovered that Indonesian drivers had the highest anger level when others shouted rudely at them, followed by random lane changing (without looking at rear view mirror) and cutting in line from surrounding drivers. However, Feng et al. [13] found that Chinese drivers often felt angry in the context of traffic obstacles, followed by illegal driving behaviors around. Smart et al. [14] found that the number of "road rage" outbreaks among drivers in Ontario, Canada increased significantly with the increase of weekly driving mileage, and the number on busy routes was significantly higher than that on fluent routes. Shinar et al. [15] proposed a frustration-aggression model to define aggressive behaviour including angry driving and found that adverse driving situations such as traffic congestion and red light waiting would make the driver feel frustrated, leading to appearance of more angry and aggressive driving behaviour. Danaf et al. [16] developed a hybrid choice-latent variable model to analyze the causes of state anger by driving simulator experiments, and the 
results indicated that state anger was significantly influenced by frustrating events and trait anger.

(2) Personal attributes factors

In addition to external situational factors, a driver's own personal attributes such as gender, age, driving years, temperament, personality, educated level and mental state can also affect anger. Gonzalez-Iglesias et al. [10] found that male drivers had a higher probability of anger outburst than female drivers, and the probability of drivers with lower educated level is higher than that of drivers with higher educated level. Lajunen et al. [6] conducted an indepth study on the relationship between aggressive behaviour, driving anger and demographic characteristics, founding that the older male drivers had lower outburst frequency of anger and aggressive behaviour, and the more miles a female driver drove per year, the lower outburst frequency of aggressive behavior. The survey conducted by Sullman et al. [17] showed that the outburst degree and frequency of anger of Malaysian drivers were negatively correlated with their age. $\mathrm{Li}$ et al. [18] found a U-shaped relationship between anger level and age for Chinese drivers, that was, below 45, driving anger level decreased with the increase of age, while over 45 , it increased with the increase of age. On the bases of statistics from improved DAS, Feng et al. [19] discovered that the anger level of Chinese drivers with A-type disposition was higher than that of drivers with B-type disposition when encountering the same incentive situation. Jovanovic et al. [20] found that the neuroticism had close relationship with aggressive driving behaviour indirectly mediated by anger, whereas the agreeableness and conscientiousness were directly correlated with aggressive driving, according to a structural equation model using the data collected from several questionnaire scales including DAS and personality inventory. Roidl et al. [21] conducted a series of driving simulator experiments and discovered that highly motivated male drivers were prone to experience more anger and further worsened their driving performance based on multiple linear regressions and path-models.

However, most of the existing studies have only analyzed the qualitative relationship between incentive situations or personal attributes and angry (emotional) driving behavior in isolation. Moreover, most research about the influencing factors of driving anger were conducted according to subjective questionnaire (scale) investigation or simulation experiments under laboratory condition where the elicited emotion is less likely to be valid as that elicited in real traffic environment, due to some demand characteristics and social desirability. Hence, the main contributions of this research are as follows: (1) a novel driving anger induction method based on naturalistic experiment was proposed and more objective data based on the experiments were collected; (2) the coupling effect of incentive situations and personal attributes on driving anger states, as well as the quantitative relationship between driving anger states with different intensity and those two kinds of influencing factors were figured out based on disaggregate theory; (3) the decisive influencing factors for driving anger were determined by sensitivity analysis based on the disaggregate theory and elasticity theory.

\section{EXPERIMENTAL DESIGNS}

\subsection{Scene Design}

In order to induce much more and real anger, a specific route including lots of busy sections across Wuchang and Hankou Districts of Wuhan was chosen for the filed experiments (see Fig. 1a). The test route is about 50.8 kilometres long which includes two expressways, two tunnels, three CBDs (central business district), 42 signalized intersections and 59 zebra crossings. The recruited subjects would frequently encounter incentive situations such as jaywalking, weaving/cutting in line, traffic jams and red light which randomly and naturally occurred, especially during morning or evening rush hours (see Fig. 1b) on the test route. Hence, 40 subjects were required to start formal experiments during the rush hours. In order to further induce more anger, the subjects would obtain extra pay if they could complete the test route ahead of reference time. It is noted that no speeding was allowed during the whole experiment. Meanwhile, to induce more real anger, the naturalistic driving experiments are designed to be single-blind, which means all subjects would complete the test route alone without any interference and they can finish the test according to their own driving style in daily life.

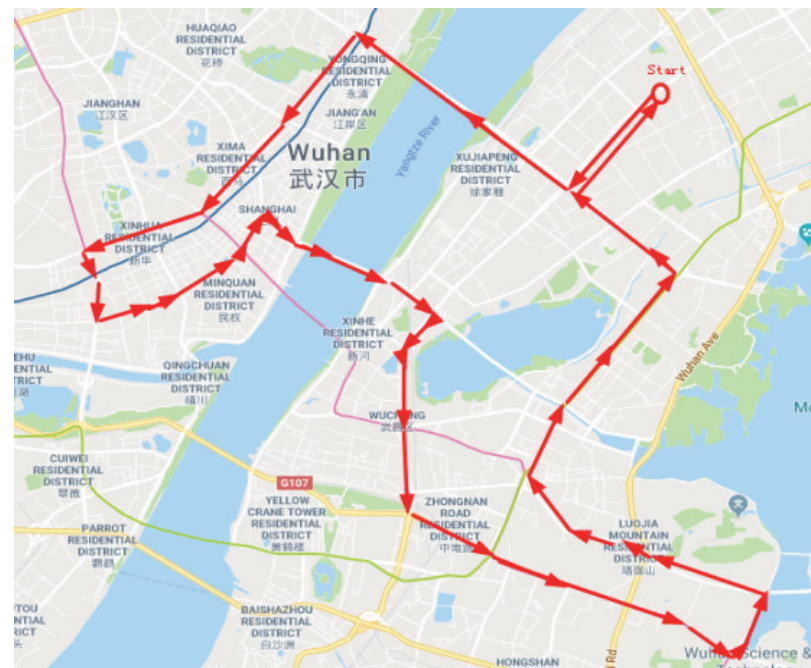

(a)The test route (shown as red line)

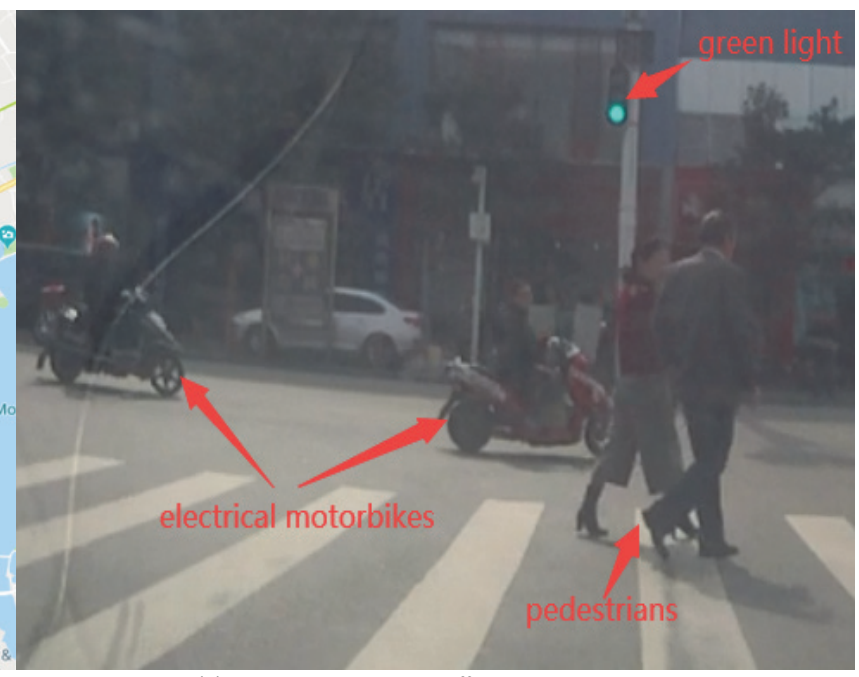

(b) Elicitation events in traffic environment

Figure 1 The test route and traffic environment of field experimental system 


\subsection{Participants}

Forty private car drivers, as the subjects for the naturalistic experiments, were recruited from Wuhan. For the sake of statistical power, more male subjects were selected because males are more apt to become angry during driving than females $[10,22]$. The average age of the subjects was $37.8 \pm 5.4$ years while their average driving age was $9.8 \pm 4$.6years. All subjects were simply checked to be in good mental condition using a POMS (profile of mood states) questionnaire, which was of importance to evaluate anger-inducing effect.

\subsection{Apparatus}

Three high-definition (HD) cameras (see Fig. 2b) were pasted on front windshield of the test vehicle (see Fig. 2a) to shoot the driver's reaction and incentive situations

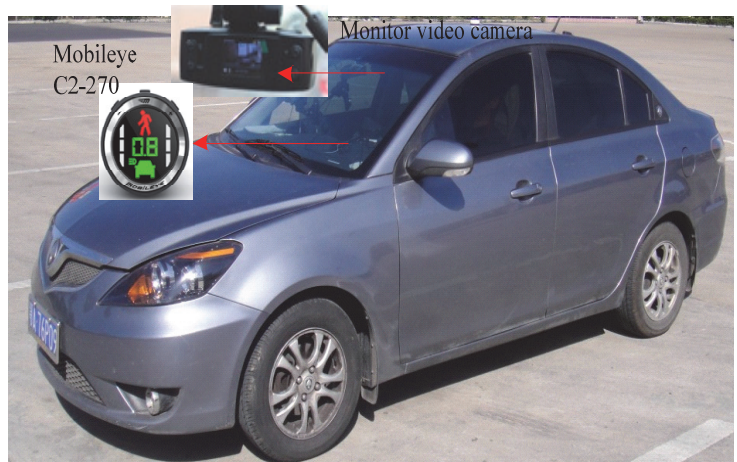

(a) The test vehicle around the vehicle. The first camera was implemented to record incentive situations around including adverse traffic environment (i.e. jam) and elicitation events (i.e. cutting in line).

The second one was used to record the driver's facial (i.e. frown) or verbal expression (i.e. name-calling) or head movement.

The third one was applied to record the driver's rough behaviors including operation frequency and fierceness amplitude of steering wheel, gear lever as well as other physical acts such as slapping steering wheel and frequent honking. Additionally, a warning system called Mobileye C2-270 was also instrumented on the test vehicle to remind the driver to keep a distance with the car ahead from perspective of safety (see Fig. 2b). Meanwhile, a clock was installed on dash board of the test car to remind the left time of the experiment for the subject.

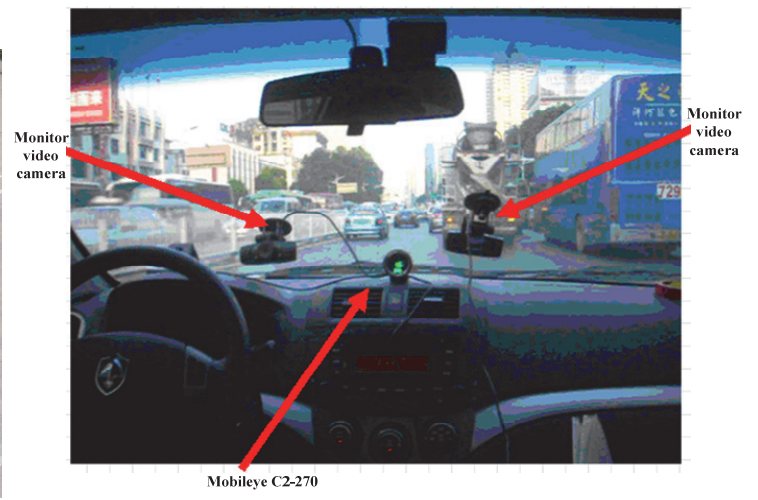

(b) HD camera systems and Mobileye C2-270

Figure 2 The test vehicle and the video shooting and warning systems

\subsection{Experiment Procedure}

(1) Experimental protocol signing. Every subject was required to sign an experimental protocol which clearly stated requirements and payment. For example, the subject had to start the formal experiments during the rush hours, namely, 8:00 a.m. or 5:00 p.m. Additionally, all subjects were forbidden to violate any traffic rules, including running red lights and speeding (speed limit is $70 \mathrm{~km} / \mathrm{h}$ ). Each subject was paid $300 \mathrm{RMB}$ (Chinese currency) for completing the experiment and they could obtain extra pay with $15 \mathrm{RMB} / \mathrm{min}$ if they could complete the test route ahead of reference time, i.e. 120 minutes, which was proved to bring a little pressure for the test on the basis of several pretests. Specially, if the subjects are not able to finish the experiment within the reference time, they can only obtain the basic pay of $300 \mathrm{RMB}$ without any deduction, according to the spirit of institutional review board (IRB) and Chinese law on scientific research. Besides, they were informed that there is no right or wrong about their reports about anger level, and they only need to report their anger level objectively and truthfully without any hiding of their true feelings in the step of self-report after experiment.

(2) Personal attributes collection. Every subject's personal attributes such as age, gender, driving years, educated level and temperament were collected by relevant questionnaire or scale. For instance, the temperament was measured by Chen Huichang Temperament Inventory [23].
(3) Adaptive driving practice. Every subject conducted 10-minute driving practice to adapt to operating performance of the test vehicle, so as to eliminate tension or discomfort when driving the test vehicle.

(4) Formal filed experiment. Every subject had to complete the test route alone without any interference, and they could finish the test in their habitual driving style. Note that all incentive situations occurring around the test vehicle, the subject's facial and verbal expression, as well as physical behaviors were automatically recorded by the HD cameras.

(5) Self-report after experiment. After finishing the whole experiment, every subject was required to recall and report his or her anger level with a ten-point scale from 0 (not at all) to 9 (very much) every two minutes during the formal experiment or any moment when an incentive situation occurred on the test route, according to the video replay from the cameras. Simultaneously, an observer with much driving experience also adopted the ten-point scale to assess the subject's anger level, in order to correct some drivers' possible subjective orientation or arbitrariness.

\section{METHODS \\ 3.1 Driving Anger Intensity Calibration}

Due to differences in temperaments, age, driving years and other personal attributes among drivers, there exist differences between the subject's self-reports and the observer's evaluation in terms of the subject's driving anger 
level. However, the two kinds of evaluation still maintain a strong similarity in reflecting the changing trend of anger level of the subjects.

For instance, Fig. 3 shows the two kinds of evaluation results for subject 5\# during the whole experiment, which indicates the two evaluations have good consistency with each other, and the probability of the two evaluations being completely consistent is even up to $38 \%(19 / 50)$. Here, as the reference time for the whole experiment is about 120 minutes, and every subject was required to report his or her anger level every two minutes or any moment when an incentive situation occurred, the average number of selfreports is 50 when deleting some invalid self-reports.

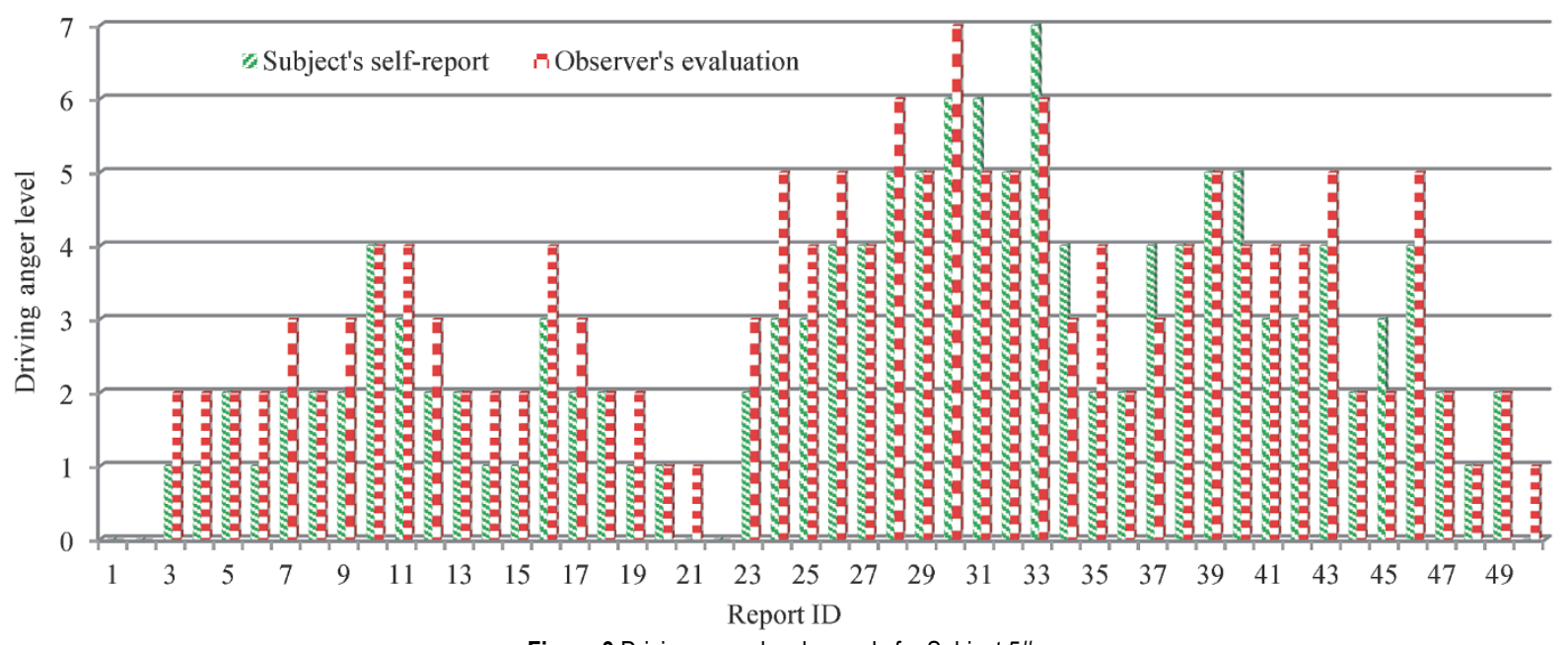

Figure 3 Driving anger level records for Subject 5\#

Moreover, the Pearson's correlation coefficients between the two evaluations for all 40 subjects were statistically analyzed, as shown in Fig. 4 . We can see that in 5 groups (ID 7, 10, 14, 23, 29) each has a correlation coefficient with more than 0.9 , while the correlation coefficient ranges between 0.7 and 0.8 from 8 groups (ID
$1,2,5,13,15,18,21,26)$. Yet, the correlation coefficient still rages between 0.8 and 0.9 from 17 groups in total. Therefore, it is concluded that the self-report about driving anger level from every subject has comparatively high degree of stability, consistency and credibility.

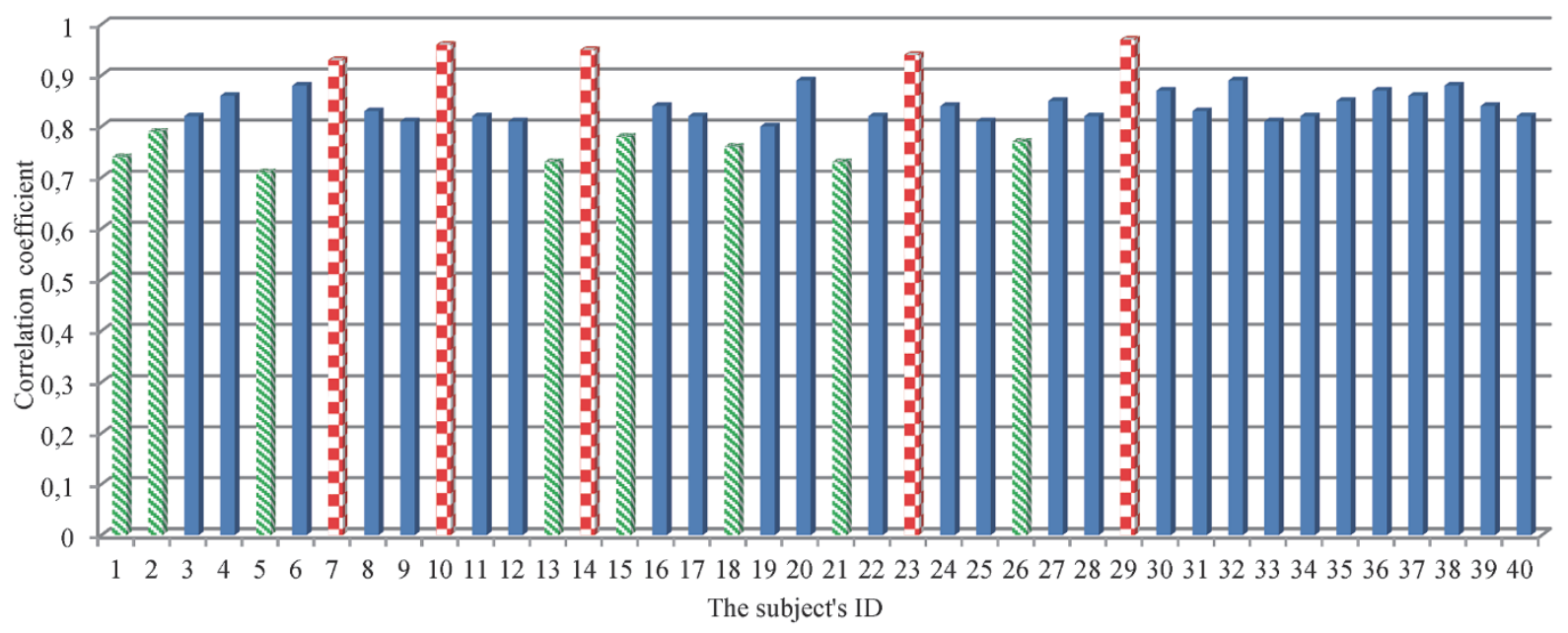

Figure 4 Correlation coefficient between self-report and observer evaluation for all subjects

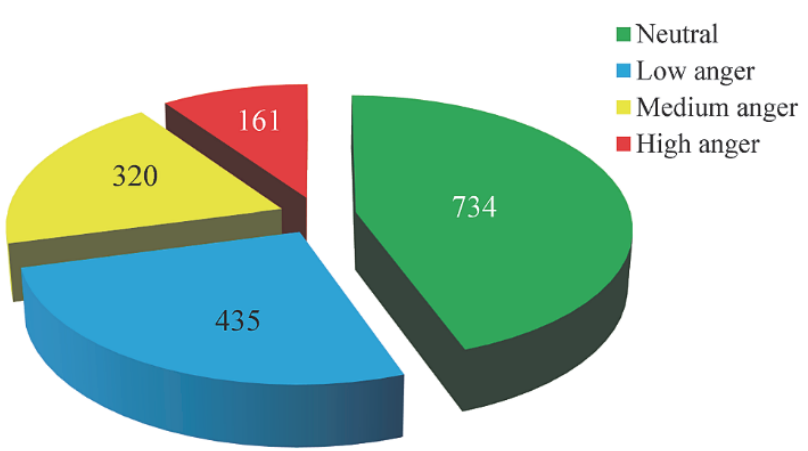

Figure 5 Instances distribution for different anger states
Then every subject's self-reported anger level was considered to be reliable, which can be used in this study. When calibrating driving anger intensity, any subject's self-report will be directly accepted if the assessment difference about anger level between the subject and the observer is smaller than 2 , or else, three more independent experts (driving years $>25$ ) in traffic behavior field will be recruited to assess the subject's anger level in line with the replayed video. And those accepted self-reports will be taken as the reference criterion for the three experts' assessments. In this study, in terms of anger intensity, all driving states were divided into four categories which included neutral state (anger level $<1$ ), low anger state $(1$ 
$\leq$ anger level $<3)$, medium anger state $(3 \leq$ anger level $<$ 5 ) and high anger state (anger level $\geq 5$ ). Therefore, 916 anger-related instances and 734 neutral (i.e. none anger) instances, were obtained for this study as shown in Fig. 5.

\subsection{Predication Model of Driving Anger Based on MNL 3.2.1 Disaggregate Theory}

It is noted that disaggregate theory has been widely applied for addressing traffic or trip issues, especially individuals' trip behavior characteristics [24, 25]. In line with disaggregate theory, every traveler is supposed to choose a specific trip mode whose utility is the best. As we know, the most vulnerable and instable part in drivervehicle-environment system is driver which makes the system more complicated because of randomness of human factors [26]. Moreover, driving anger, as a common human factor, has negative influence on traffic safety nowadays. It is well known that incentive situations and personal attributes can directly or indirectly induce driving anger. Hence, driving anger will emerge with a certain discreteness and nonlinearity due to the influencing factors. The nature of whether a person has driving anger or not can be regarded as a result of behavior selection. Under the action of various influencing factors, the person will choose a specific type or grade of driving anger based on personal maximum expectation utility. Fortunately, the disaggregate model aforementioned is feasible to quantitatively depict the couple effect of multiple influencing factors on driving anger because of its good adaptability.

In this study, Multinomial logit (MNL) model based on disaggregate theory is proposed to analyze the sensitivity of incentive situations and personal attributes to driving anger. Here, the utility function consists of a fixed item and a random item. For instance, the utility function of the $j$-th trip mode chosen by the $m$-th traveler can be expressed as the following:

$U_{j m}=V_{j m}+\varepsilon_{j m}$

$V_{j m}=\sum_{k=1}^{K} \lambda_{k} X_{j m k}$

where, $V_{j m}, \varepsilon_{j m}$ are the fixed item and the random item of the utility function for the $j$-th trip mode chosen by the $m$ th traveller, respectively. $X_{j m k}$ is the value of the $k$-th attribute (i.e. influencing factor) of the $j$-th trip mode chosen by the $m$-th traveler; $\lambda_{k}$ is the corresponding estimation value of $X_{j m k}$ and $K$ is the total number of the attributes which contribute to the $j$-th trip mode. For the above disaggregate model, Logit model and Probit model can be established when $\varepsilon_{j m}$ belongs to double exponential distribution and multi-dimensional normal distribution, respectively.

\subsubsection{Model Construction Based on MNL}

As multinomial logit (MNL) model based on disaggregate theory is widely used in traffic fields due to its simple mathematical forms whose physical significance is easy to understand, a prediction model of driving anger based on MNL is proposed in this study. Assuming that $\varepsilon_{j m}$ and $V_{j m}$ in Eq. (1) is independent of each other, and the $\varepsilon_{j m}$ obey the Gumbel distribution, then the disaggregation model consisting of Eq. (1) and Eq. (2) can be transformed into MNL model according to the following process. It is assumed that the distribution function and density function of $\varepsilon_{j m}$ can be respectively expressed as follows [27]:

$$
\begin{aligned}
& F(\varepsilon)=\mathrm{e}^{-\lambda \mathrm{e}^{-\varepsilon}} \\
& f(\varepsilon)=\lambda \mathrm{e}^{-\varepsilon} \mathrm{e}^{-\lambda \mathrm{e}^{-\varepsilon}}
\end{aligned}
$$

In Eq. (3) and Eq. (4), $\lambda>0$, according to utility theory, set the random vector $\varepsilon_{n}=\left(\varepsilon_{1 n}, \varepsilon_{2 n}, \ldots, \varepsilon_{j m}\right)$. The joint distribution function and the joint density function of $\varepsilon_{m}$ are $F\left(\varepsilon_{1 m}, \varepsilon_{2 m}, \ldots, \varepsilon_{j m}\right), f\left(\varepsilon_{1 n}, \varepsilon_{2 n}, \ldots, \varepsilon_{j m}\right)$, respectively. The basic form of MNL model can be deduced as follows:

$$
\begin{aligned}
P_{j m} & =\operatorname{Prob}\left(U_{j m}>U_{i m}, \forall j \neq i\right) \\
& =\operatorname{Prob}\left(V_{j m}+\varepsilon_{j m}>V_{i m}+\varepsilon_{i m}, \forall j \neq i\right) \\
& =\operatorname{Prob}\left(\varepsilon_{i m}<V_{j m}+\varepsilon_{j m}-V_{i m}, j \neq i, \forall i \in A_{m}\right)
\end{aligned}
$$

where, $A_{m}$ is the set of all possible travel modes chosen by traveler $m$, the probability of the $m$-th traveler choosing the first travel mode is expressed by Eq. (6):

$$
\begin{aligned}
& P_{1 m}=\int_{-\infty}^{+\infty} \int_{-\infty}^{V_{1 m}-V_{2 m}+\varepsilon_{1 m}} \ldots \\
& \int_{-\infty}^{V_{1 m}-V_{2 m}+\varepsilon_{1 m}} f\left(\varepsilon_{1 m}, \varepsilon_{2 m}, \ldots, \varepsilon_{i m}\right) \mathrm{d} \varepsilon_{1 m}, \mathrm{~d} \varepsilon_{2 m}, \ldots, \mathrm{d} \varepsilon_{i m}
\end{aligned}
$$

Substituting Eqs. (3) and (4) into Eq. (6), we can get the probability that the $m$-th traveler chooses the $j$-th travel mode as follows:

$$
\begin{aligned}
& P_{j m}=\int_{-\infty}^{+\infty} \Pi \exp \left\{-\lambda \exp \left[-V_{j m}-V_{i m}+\varepsilon\right]\right\} \times \\
& \times \lambda \mathrm{e}^{-\varepsilon} \exp \left(-\lambda \mathrm{e}^{-\varepsilon}\right) \mathrm{d} \varepsilon \\
& =\int_{-\infty}^{+\infty} \Pi \exp \left\{-\lambda \exp \left[-V_{j m}-V_{i m}+\varepsilon\right]\right\} \times \lambda \mathrm{e}^{-\varepsilon} \mathrm{d} \varepsilon \\
& =\int_{-\infty}^{+\infty} \exp \left\{-\lambda \mathrm{e}^{-\varepsilon} \sum_{i} \exp \left[V_{i m}-V_{j m}\right]\right\} \times \lambda \mathrm{e}^{-\varepsilon} \mathrm{d} \varepsilon \\
& =\frac{\mathrm{e}^{V_{j m}}}{\sum_{i=1}^{I_{m}} \mathrm{e}^{V_{i m}}}
\end{aligned}
$$

where, $i$ and $j$ represent the sequence number of travel modes, it particularly refers to the sequence number of driving anger intensity divided in this paper; $I_{m}$ represents the total number of driving anger intensity divided, and 0 $\leq P_{j m} \leq 1 \sum_{j \in A_{m}} P_{j m}=1$.

In practical application, the maximum likelihood estimation method combined with Newton-Raphson method can be used to solve the MNL model [27]. 


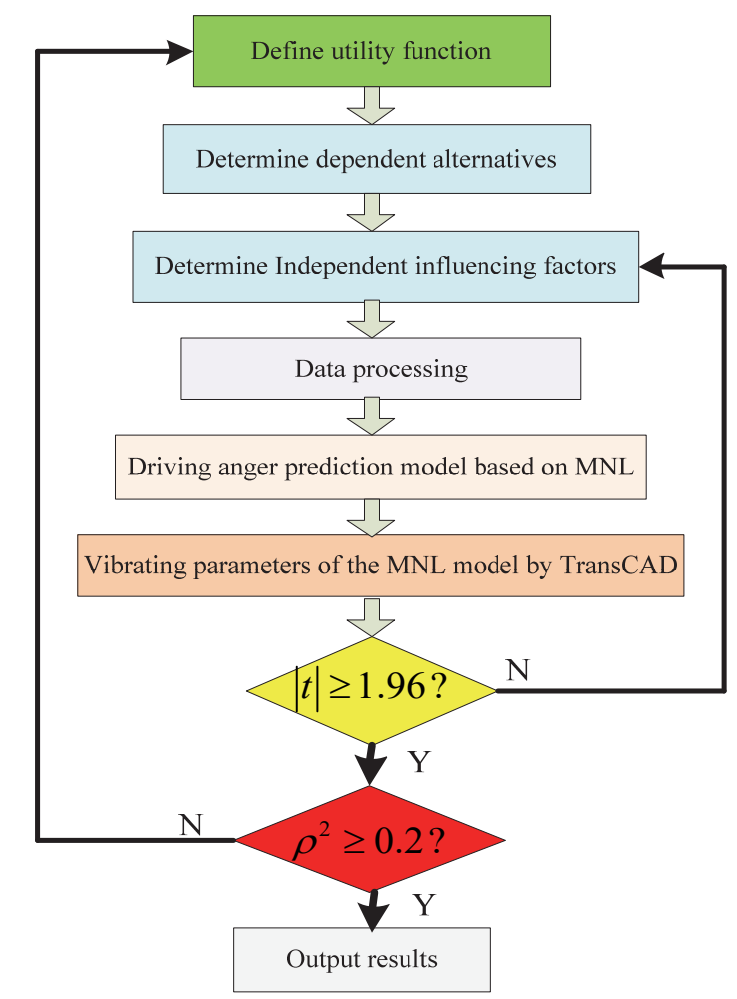

Figure 6 Constructing process of prediction model of driving anger based on MNL
Therefore, a prediction model of driving anger states with different intensity based on MNL is constructed with the processes shown in Fig. 6, where, $t$ is the test value of variable parameter $\lambda$, and $\rho^{2}$ is McFadden determination coefficient of the proposed model, which is often used to evaluate the model's effectiveness (i.e. fitting degree).

\subsubsection{Definition of Independent Variables and Dependent Variables}

As above-mentioned, all driving anger states are divided into four categories consisting of neutral, low anger, medium anger and high anger. Correspondingly, the four driving anger states are considered to be the alternatives (i.e. dependent variables) of the MNL-based model proposed in this study, and they are denoted by $A, B$, $C$ and $D$, respectively. The definition of the alternatives is indicated in Tab. 1.

Considering different inducing sources of driving anger, the influencing factors of driving anger are divided into two categories which include personal attributes and incentive situations. All the influencing factors are independent variables of the proposed model. The definitions and descriptions of the influencing factors are demonstrated in Tab. 2.

Table 1 Definition and description of dependent variable (alternatives) of the proposed model

\begin{tabular}{|c|c|}
\hline Driving anger states & Definition and description \\
\hline $\begin{array}{l}\text { None anger (Neutral) } \\
\text { (Denoted by A) }\end{array}$ & $\begin{array}{c}\text { Anger level }<1 \\
\text { (Calm and relaxed, no abnormal facial or verbal expression) }\end{array}$ \\
\hline $\begin{array}{c}\text { Low anger } \\
\text { (Denoted by B) }\end{array}$ & $\begin{array}{c}1 \leq \text { Anger level }<3 \\
\text { (Slight verbal or physical aggression like cursing or abusing others in car, slapping the steering wheel) }\end{array}$ \\
\hline $\begin{array}{l}\text { Medium anger } \\
\text { (Denoted by C) }\end{array}$ & $\begin{array}{c}3 \leq \text { Anger level }<5 \\
\text { (More severe verbal or physical attack such as shaking the car window off for name-calling, or making hostile gestures like the } \\
\text { middle finger, rapid honking. }\end{array}$ \\
\hline $\begin{array}{c}\text { High anger } \\
\text { (Denoted by D) }\end{array}$ & $\begin{array}{c}\text { Anger level } \geq 5 \\
\text { (Frequent honking, cutting in line forcedly, overtaking a car forcedly, intentionally block others and other more dangerous or } \\
\text { offensive behaviors. }\end{array}$ \\
\hline
\end{tabular}

Table 2 The description and definition of independent variables of the proposed model

\begin{tabular}{|c|c|c|c|}
\hline \multicolumn{2}{|r|}{ Influencing factors } & Variables & Description and definition \\
\hline \multirow{7}{*}{$\begin{array}{l}\text { Personal } \\
\text { attributes }\end{array}$} & Gender & $x_{1}$ & male- 1 , female- 0 \\
\hline & Age & $x_{2}$ & actual age \\
\hline & Driving experience & $x_{3}$ & actual driving years \\
\hline & Educated level & $x_{4}$ & $\begin{array}{c}\text { primary school and below-0, junior middle school-1, senior middle school-2, } \\
\text { graduate and above-3 }\end{array}$ \\
\hline & Temperament & $x_{5}$ & melancholic-0, phlegmatic-1, sanguineous-2, choleric-3 \\
\hline & Whether a professional driver & $x_{6}$ & yes- 1 , no-0 \\
\hline & $\begin{array}{l}\text { Whether involved in traffic } \\
\text { accident }\end{array}$ & $x_{7}$ & yes-1, no-0 \\
\hline \multirow{6}{*}{$\begin{array}{l}\text { Incentive } \\
\text { situations }\end{array}$} & Weather & $x_{8}$ & sunny-0,cloudy-1,rainy-2 \\
\hline & $\begin{array}{l}\text { Traffic facilities(road surface, } \\
\text { traffic sign) condition }\end{array}$ & $x_{9}$ & good- 0 , average- 1 , bad- 2 \\
\hline & Traffic congestion level & $x_{10}$ & fluent- 0 , slightly congested- 1 , congested- 2 , seriously congested- 3 \\
\hline & Red light waiting & $x_{11}$ & $\operatorname{short}(<20 \mathrm{~s})-0$, comparatively long $(20-40 \mathrm{~s})-1$, long $(40-60 \mathrm{~s})-2$, too long $(60 \mathrm{~s}+)-3$ \\
\hline & Time pressure felt & $x_{12}$ & none-0,small-1, medium-2, high-3 \\
\hline & Behaviors of road user surround & $x_{13}$ & $\begin{array}{c}\text { civilized behavior-0, slowly driving car ahead/oversized vehicle ahead-1, } \\
\text { Jaywalking/cyclist crossing/ merging arbitrarily of non-motor vehicle }-2 \text {, other } \\
\text { vehicle' weaving/cutting in line forcedly-3 }\end{array}$ \\
\hline
\end{tabular}

\subsection{Calibration and Analysis of Model Parameters}

As illustrated in Fig. 6, after the MNL model with data source of the alternatives and the influencing factors was constructed in TransCAD software, the parameters of the proposed model thus can be calibrated according to the function of Multinomial Logit Estimation embedded in the software. The results of parameters calibration is shown in Tab. 3.

According to statistics theory, an independent variable is verified to have significant impact on the alternatives of the proposed model if absolute value of $t$ is not less than 1.96 when belief degree of the impact of the variable on the alternative is set to be $95 \%$, besides the correctness of sign of the variable. As indicated in Tab. 3, the absolute values 
of $t$ of all variables are bigger than 1.96, which demonstrates that the 13 selected personal attributes have significant impact on driving anger states. Note that a model can fit the actual data well in practical application if $\rho^{2} \in[0.2,0.4]$ according to literature [27].The parameters calibration results show that the value of McFadden determination coefficient $\left(\rho^{2}\right)$ of the proposed model is 0.3672 , implying that the proposed model is effective to predict driving anger states.

Table 3 Estimation results of the parameters of the proposed model

\begin{tabular}{|c|c|c|c|c|}
\hline Influencing factors & Variable & $\lambda_{k}$ & Std of $\lambda_{k}$ & $t$ \\
\hline Gender & $x_{1}$ & 0.8426 & 0.2362 & 3.2726 \\
\hline Age & $x_{2}$ & 0.0517 & 0.0081 & 6.9342 \\
\hline Educated level & $x_{3}$ & 0.4024 & 0.1386 & 2.5713 \\
\hline Driving years & $x_{4}$ & -0.0884 & 0.0374 & -3.0272 \\
\hline Temperament & $x_{5}$ & 1.8692 & 0.0967 & 3.5839 \\
\hline Whether a professional driver & $x_{6}$ & -0.4685 & 0.0968 & 1.6612 \\
\hline Whether involved in traffic accident & $x_{7}$ & 0.9513 & 0.1898 & 4.2726 \\
\hline Weather & $x_{8}$ & 0.6776 & 0.2415 & 3.8824 \\
\hline Traffic facilities condition & $x_{9}$ & 0.7565 & 0.0482 & 4.0335 \\
\hline Traffic congestion level & $x_{10}$ & 0.6974 & 0.1306 & 6.1723 \\
\hline Red light waiting & $x_{11}$ & 0.5506 & 0.2694 & 4.8551 \\
\hline Pressure of time & $x_{12}$ & 1.1679 & 0.1983 & 4.6334 \\
\hline Road user behaviors & $x_{13}$ & 1.5778 & 0.2159 & 7.2726 \\
\hline
\end{tabular}

\section{RESULTS}

\subsection{Unity Function for Driving Anger States}

According to aforementioned disaggregate theory and parameter calibration results of the MNL-based model, we can obtain the variable and its parameter for the corresponding unity function of each driving anger state (i.e. $A, B, C, D)$, which are shown in Tab. 4.

According to Tab. 4, the utility function $V_{A}, V_{B}, V_{C}$ and $V_{D}$, corresponding to the four driving anger states $A, B, C$ and $D$, respectively, can be expressed as follows:

Table 4 The variables' parameter for different driving anger states

\begin{tabular}{|c|c|c|c|c|c|}
\hline \multirow{2}{*}{ Impact factors } & \multirow{2}{*}{ Variable } & \multicolumn{4}{|c|}{ The variables' parameters } \\
\hline & & $A$ & $B$ & $C$ & $D$ \\
\hline Gender & $x_{1}$ & & 0.8426 & 0.8426 & \\
\hline Age & $x_{2}$ & 0.0517 & & & 0.0517 \\
\hline Education level & $x_{3}$ & 0.4024 & 0.4024 & 0.4024 & \\
\hline Driving experience & $x_{4}$ & & -0.0884 & -0.0884 & -0.0884 \\
\hline Temperament & $x_{5}$ & 1.8712 & 1.8712 & 1.8712 & 1.8712 \\
\hline Whether a professional driver & $x_{6}$ & -0.4685 & -0.4685 & & \\
\hline Whether involved in traffic accident & $x_{7}$ & 0.9513 & & 0.9513 & 0.9513 \\
\hline Weather & $x_{8}$ & & & 0.6776 & 0.6776 \\
\hline Traffic facilities condition & $x_{9}$ & & & & 0.7485 \\
\hline Traffic congestion level & $x_{10}$ & & 0.6974 & 0.6974 & 0.6974 \\
\hline Red light waiting & $x_{11}$ & & & & 0.5506 \\
\hline Pressure of time & $x_{12}$ & 1.1593 & 1.1593 & 1.1593 & 1.1593 \\
\hline Road user behaviors & $x_{13}$ & & 1.5686 & 1.5686 & 1.5686 \\
\hline
\end{tabular}

$V_{A}=0.0517 x_{2}+0.4024 x_{3}+1.8712 x_{5}-0.4685 x_{6}+$

$+0.9513 x_{7}+1.1593 x_{12}$

$V_{B}=0.8426 x_{1}+0.4024 x_{3}-0.0884 x_{4}+1.8712 x_{5}-$ $-0.4685 x_{6}+0.6974 x_{10}+1.1593 x_{12}+1.5686 x_{13}$

$V_{C}=0.8426 x_{1}+0.4024 x_{3}-0.0884 x_{4}+1.8712 x_{5}$ $+0.9513 x_{7}+0.6776 x_{8}+0.6974 x_{10}+1.1593 x_{12}+$

$+1.5686 x_{13}$

$V_{D}=0.0517 x_{2}-0.0884 x_{4}+1.8712 x_{5}+0.9513 x_{7}$ $+0.6776 x_{8}+0.7485 x_{9}+0.6974 x_{10}+0.5506 x_{11}+$

$+1.1593 x_{12}+1.5686 x_{13}$

\subsection{Sensitivity Analysis of Influencing Factors}

In order to test the impact of the influencing factors on the occurrence of a certain driving anger state with a specific intensity, a concept called elasticity, originated from price theory of microscopic economics, was introduced to conduct sensitivity analysis for the influencing factors. Elasticity is a special indictor which can reflect the variation of a variable along with the change of another variable, when the two variables are correlated with each other from the perspective of statistics. Thus, the elasticity value is a relative value, expressed by the ratio of change rate for the two variables. Correspondingly, the elasticity value of the $m$-th driver's change scope of the probability for selecting the $j$-th scheme when the $k$-th impact factor varies, can be calculated as follows in terms of disaggregate theory.

$$
E_{X_{j m}^{j m}}^{p}=\lambda_{k} \overline{X_{j m k}}\left(1-P_{j m}\right)
$$

where, $\overline{X_{j m k}}$ is the average value of the $k$-th attribute for all the drivers who presented the $j$-th anger states.The elasticity value is often expressed in the form of absolute value. When the absolute value is less than 1 , it demonstrates that the influencing factor is short of 
elasticity to the occurrence of driving anger, because the change rate of the probability of a driving anger state is less than that of the corresponding influencing factors. Otherwise, it indicates that the influencing factor is rich in elasticity to occurrence of driving anger.

\subsubsection{Sensitivity Analysis of Personal Attributes}

Firstly, as shown in Tab. 5, the estimation parameters of the proposed model for gender, age, educated level, driving years, temperament, whether a professional driver or not and whether involved in traffic accident or not are $0.838,0.048,0.396,-0.084,1.864,-0.464$ and 0.946 , respectively. Secondly, after $P_{j m}$ for the corresponding driving anger state is obtained through Eq. (7), we can calculate the elasticity value of an influencing factor for driving anger by Eq. (12), with the results shown in Tab. 5. We can see that the average elasticity values of age and temperament for none anger (neutral), low anger, medium anger, high anger are $1.254,2.713$, respectively, meaning that the two factors are decisive for emerging anger while driving when encountering incentive situations in real traffic environments.

\section{(1) Gender}

As illustrated in Tab. 5, the average value of gender for neutral state $(A)$ is smaller than 0.5 , while higher than 0.5 for other driving anger states, showing more male drivers are likely to become angry when suffering the same incentive situations in real traffic environment. Moreover, the proportion of male drivers increases with the increment of anger intensity, which further implies the male drivers are inclined to become angrier than females if meeting the same incentive situations. The result is in line with the finding in literature [10]. Besides, according to Tab. 5, it is noted that the elasticity value $\left(E_{1}\right)$ of gender for all driving anger states is smaller than 1 , which indicates that gender is short of elasticity to the occurrence of driving anger. It reveals that gender can influence driving anger state, but without significant sensitivity.

(2) Age

As indicated in Tab. 5, the average age of the subjects presenting neutral state reaches up to 41.3 , while the average age of the subjects presenting high anger state is 28.5. Moreover, with the increase of driving anger intensity, the average age of the subjects decreases, which reveals that the younger subjects are more prone to become angry while driving when facing incentive situations in real traffic environment. Consistently, Herrero-Fernández et al [28] found that younger drivers scored higher than older ones in all aggressive behaviors which have strong correlation with anger. Further, it is found that the elasticity value $\left(E_{2}\right)$ of age for all driving anger states is bigger than 1 , which demonstrates that age is rich in elasticity to the occurrence of driving anger, implying that age can be determined as one of the most important factors to predict driving anger.

\section{(3) Educated level}

As shown in Tab. 5, anger intensity increases along with the increase of educated level of the subjects, which may have a deviation with our common sense. Vujanic et al. [29] found that drivers with high educated level, have better psychophysical characteristics including general intelligence, concentration level and stress tolerance, with regard to driving safety. Note that the investigated subjects presenting anger states, especially medium and high anger were born in the 1980 s or 1990 s, which means that they generally received a comparably good education due to economic development of China during their growth. We can see that the elasticity value $\left(E_{3}\right)$ of educated level for all driving anger states is smaller than 1 , which indicates that educated level is short of elasticity to the occurrence of driving anger, meaning that educated level cannot be considered to be a decisive factor for emerging anger while driving.

\section{(4) Driving years (experience)}

As we know, driving years are strongly associated with a driver's driving skill or psychological state which finally influences his or her driving behavior. As illustrated in Tab.5, the average driving years decrease dramatically with the increase of driving anger intensity. Particularly, the average driving years for high anger state is only 3.5 years. Coincidently, the previous research also found that a traffic accident usually happens with an average driving experience of 3 years [30]. The reason may be that the accident-related driver has good driving skill, but an arrogant psychology and easily triggered anger when facing incentive situations, leading to low safety awareness when he or she has driving experience of 3 years. Furthermore, the absolute elasticity value $\left(\left|E_{4}\right|\right)$ of driving years for all anger states is smaller than 1 , which indicates that driving years is short of elasticity to the occurrence of driving anger, meaning that driving years cannot be considered to be a decisive factor for emerging anger while driving.

\section{(5) Temperament}

As indicated in Tab. 5, the average value of temperament increases with driving anger intensity. In other words, the driving anger intensity will markedly grow when a subject's temperament changes from melancholic, phlegmatic, sanguineous to choleric, in this order. More subjects with phlegmatic temperament were in neutral and low anger state while more subjects with choleric temperaments were in high anger state. It can be further learned from Tab. 5 that the elasticity values $\left(E_{5}\right)$ of temperament for all anger states are greater than 1 , indicating that temperament is rich in elasticity to the occurrence of driving anger, that is, temperament is a decisive factor for emerging anger while driving. The elasticity values of the four driving anger states are quite different. Particularly, the elasticity value of temperament for high anger was three times that of neutral state, indicating that the possibility of high anger induced was three times that of neutral states when the driver with choleric temperament encountered incentive situations in real traffic environments.

\section{(6) Whether a professional driver or not}

As indicated in Tab. 5, it is found that average value of whether a professional driver or not for neutral and low anger state is greater than 0.5 , while smaller than 0.5 for medium and high anger states. It indicates that professional drivers can better regulate and control their emotions than non-professional drivers when encountering incentive situations. Additionally, the proportion of non-professional drivers increased with the increase of anger intensity. It is further shown from Tab. 5 that the elasticity values $\left(E_{6}\right)$ of whether a professional driver or not for all anger states are 
less than 1 , indicating that the influencing factor is short in elasticity to the occurrence of driving anger. Therefore, whether a professional driver or not does influence driver anger state to some extent, yet, it cannot be considered a decisive factor for emerging anger while driving.

\section{(7) Whether involved in an accident or not}

According to accident tendency theory, some drivers are prone to be involved in a traffic accident because of their unique psychological characteristics. And related research [21] shows that drivers with anger traits are more likely to make driving mistakes and errors due to their limited perception and decision-making ability, leading to traffic accidents finally. Therefore, drivers who have had traffic accidents may possess a greater chance to own anger traits than those who have not. Moreover, drivers with anger trait are more likely to become angry when encountering the incentive situations in real traffic environment. As shown in Tab. 5, although the average values of whether involved in an accident or not for all driving anger states are smaller than 0.5 , the proportion of drivers with accident involvement still grows with the increase of driving anger intensity. The elasticity values $\left(E_{7}\right)$ of the influencing factor for all anger states are less than 1 , showing that the factor is short in elasticity to the occurrence of driving anger, which means the factor is not a decisive factor for inducing driving anger. Additionally, the elasticity value corresponding to high anger is significantly bigger than that of other anger states, which indicates that drivers involved in a traffic accident are more possible to produce high anger than other anger states when meeting the incentive situations.

Table 5 The elasticity value of personal attributes

\begin{tabular}{|c|c|c|c|c|c|c|c|}
\hline \multirow[b]{2}{*}{ Driving anger state } & \multirow[b]{2}{*}{ Choice probability } & \multicolumn{3}{|c|}{ Gender } & \multicolumn{3}{|c|}{ Age } \\
\hline & & $\lambda_{1}$ & $\overline{\overline{X_{1}}}$ & $E_{1}$ & $\lambda_{2}$ & $\overline{\overline{X_{2}}}$ & $E_{2}$ \\
\hline A & 0.425 & 0.843 & 0.478 & 0.281 & 0.052 & 41.872 & 1.362 \\
\hline B & 0.254 & 0.843 & 0.568 & 0.356 & 0.052 & 38.215 & 1.278 \\
\hline $\mathrm{C}$ & 0.213 & 0.843 & 0.629 & 0.415 & 0.052 & 33.243 & 1.182 \\
\hline D & 0.108 & 0.843 & 0.708 & 0.483 & 0.052 & 28.846 & 1.194 \\
\hline \multirow[b]{2}{*}{ Driving anger state } & \multirow[b]{2}{*}{ Choice probability } & \multicolumn{3}{|c|}{ Educated level } & \multicolumn{3}{|c|}{ Driving years } \\
\hline & & $\lambda_{3}$ & $\overline{X_{3}}$ & $E_{3}$ & $\lambda_{4}$ & $\overline{X_{4}}$ & $E_{4}$ \\
\hline$A$ & 0.425 & 0.402 & 1.986 & 0.574 & -0.088 & 12.262 & -0.865 \\
\hline$B$ & 0.254 & 0.402 & 2.225 & 0.663 & -0.088 & 10.167 & -0.776 \\
\hline$C$ & 0.213 & 0.402 & 2.478 & 0.742 & -0.088 & 6.278 & -0.712 \\
\hline$D$ & 0.108 & 0.402 & 2.627 & 0.898 & -0.088 & 3.436 & -0.688 \\
\hline \multirow[b]{2}{*}{ Driving anger state } & \multirow[b]{2}{*}{ Choice probability } & \multicolumn{3}{|c|}{ Temperament } & \multicolumn{3}{|c|}{ Whether a professional driver or not } \\
\hline & & $\lambda_{5}$ & $\overline{X_{5}}$ & $E_{5}$ & $\lambda_{6}$ & $\overline{X_{6}}$ & $E_{6}$ \\
\hline$A$ & 0.425 & 1.871 & 0.864 & 1.164 & -0.469 & 0.485 & -0.142 \\
\hline$B$ & 0.254 & 1.871 & 1.505 & 2.142 & -0.469 & 0.514 & -0.171 \\
\hline$C$ & 0.213 & 1.871 & 2.236 & 3.318 & -0.469 & 0.464 & -0.178 \\
\hline$D$ & 0.108 & 1.871 & 2.614 & 4.228 & $\begin{array}{l}-0.469 \\
\end{array}$ & 0.437 & -0.196 \\
\hline \multirow[b]{2}{*}{ Driving anger state } & \multirow[b]{2}{*}{ Choice probability } & \multicolumn{3}{|c|}{ Whether involved an accident or not } & & & \\
\hline & & $\lambda_{7}$ & $\overline{\overline{X_{7}}}$ & $E_{7}$ & & & \\
\hline$A$ & 0.425 & 0.951 & 0.304 & 0.238 & & & \\
\hline$B$ & 0.254 & 0.951 & 0.325 & 0.219 & & & \\
\hline$C$ & 0.213 & 0.951 & 0.426 & 0.261 & & & \\
\hline$D$ & 0.108 & 0.951 & 0.516 & 0.374 & & & \\
\hline
\end{tabular}

\subsubsection{Sensitivity Analysis of Incentive Situations}

According to the data collected from the field experiments, $X_{j m k}$ can be calculated on the basis of the average value of the incentive situations for all the drivers who selected the ith anger state. Then, after $P_{j m}$ for the corresponding driving anger state is obtained through Eq. (7), we can calculate the elasticity value of an incentive situation for driving anger by Eq. (12), with the results shown in Tab. 6. The impact of various incentive situations on driving anger states can be obtained according to Tab. 6. We can see that the average elasticity value of illegal behaviors from people around for none anger (neutral), low anger, medium anger, high anger is 2.914, meaning that the factor is decisive for emerging anger while driving.

\section{(1) Weather}

As illustrated in Tab. 6, average value of weather for neutral state is less than 0.5 , and the values for low or medium anger state are both between 0.5 and 1 , while the value for high anger is slightly greater than 1 . It shows that driving neutral state mostly occurs in sunny days, and low or medium anger states mostly appear in cloudy days while high anger states tend to occur on rainy days. In other words, the drivers are likely to become angrier in more adverse weather. The conclusion can provide theoretical support for adding relevant training items in adverse weather for driving license test, or reminding the drivers to keep calm state in adverse weather from the perspective of traffic management authority. In addition, the elasticity values (E8) of weather for all driving states are less than 1, which means that weather change is short in elasticity to the occurrence of driving anger, i.e. the impact of weather change on outburst possibility of driving anger is small. Hence, weather is not a decisive factor for inducing driving anger. However, the elastic value of weather corresponding to high anger was 0.681 , significantly higher than that of other three driving states, which indicates that the influence of weather change on the inducing probability of high anger state was greater than other driving states.

\section{(2) Traffic facilities condition}

As we know, if the road surface is not smooth or damaged, or traffic signs are not clear, or crosswalks are 
set unreasonably, we are prone to generate some negative emotions like anger or stress while driving. From Tab. 6, we can find a driver's anger intensity increases with the deterioration of traffic conditions. The conclusion can provide some references for the design and maintenance of traffic facilities. Besides, the elasticity values (E9) of traffic facilities condition for all driving states are smaller than 1 , which means traffic facilities condition is not a decisive factor for inducing driving anger. However, the elastic value of traffic facilities condition for high anger is 0.974 , which is $1.5 \sim 2.5$ times that of other three driving states, indicating that the probability of average or bad facilities condition for inducing high anger state was 1.5 2.5 times that of other three driving states.

Table 6 The elasticity value of incentive situations

\begin{tabular}{|c|c|c|c|c|c|c|c|}
\hline \multirow{2}{*}{ Driving anger state } & \multirow{2}{*}{ Choice probability } & \multicolumn{3}{|c|}{ Weather } & \multicolumn{3}{|c|}{ Traffic facilities condition } \\
\hline & & $\lambda_{8}$ & $\overline{X_{8}}$ & $E_{8}$ & $\lambda_{9}$ & $\overline{X_{9}}$ & $E_{9}$ \\
\hline$A$ & 0.425 & 0.678 & 0.417 & 0.183 & 0.749 & 0.756 & 0.389 \\
\hline$B$ & 0.254 & 0.678 & 0.668 & 0.317 & 0.749 & 0.948 & 0.523 \\
\hline$C$ & 0.213 & 0.678 & 0.851 & 0.426 & 0.749 & 1.196 & 0.712 \\
\hline$D$ & 0.108 & 0.678 & 1.172 & 0.681 & 0.749 & 1.554 & 0.974 \\
\hline \multirow{2}{*}{ Driving anger state } & \multirow{2}{*}{ Choice probability } & \multicolumn{3}{|c|}{ Traffic congestion level } & \multicolumn{3}{|c|}{ Red light waiting } \\
\hline & & $\lambda_{10}$ & $\overline{X_{10}}$ & $E_{10}$ & $\lambda_{11}$ & $\overline{X_{11}}$ & $E_{11}$ \\
\hline$A$ & 0.425 & 0.697 & 0.518 & 0.425 & 0.551 & 0.914 & 0.412 \\
\hline$B$ & 0.254 & 0.697 & 0.986 & 0.592 & 0.551 & 1.514 & 0.624 \\
\hline$C$ & 0.213 & 0.697 & 1.623 & 0.523 & 0.551 & 1.803 & 0.791 \\
\hline$D$ & 0.108 & 0.697 & 2.264 & 0.864 & 0.551 & 2.238 & 0.914 \\
\hline \multirow{2}{*}{ Driving anger state } & \multirow{2}{*}{ Choice probability } & \multicolumn{3}{|c|}{ Pressure of time } & \multicolumn{3}{|c|}{ Illegal behaviors from people around } \\
\hline & & $\lambda_{12}$ & $\overline{X_{12}}$ & $E_{12}$ & $\lambda_{13}$ & $\overline{X_{13}}$ & $E_{13}$ \\
\hline$A$ & 0.425 & 1.159 & 1.226 & 0.414 & 1.569 & 1.357 & 1.428 \\
\hline$B$ & 0.254 & 1.159 & 1.924 & 0.672 & 1.569 & 1.772 & 1.898 \\
\hline$C$ & 0.213 & 1.159 & 2.236 & 0.793 & 1.569 & 2.44 & 3.341 \\
\hline$D$ & 0.108 & 1.159 & 2.545 & 0.965 & 1.569 & 2.785 & 4.987 \\
\hline
\end{tabular}

\section{(3) Traffic congestion}

It is well known that the pace of urban life and work is fast nowadays. Once traffic congestion occurs, drivers are more likely to breed negative emotions such as anxiety or irritation. As shown in Tab. 6, when the average traffic congestion level is 0.486 , that is, the traffic state is between unblocked and light congestion, drivers are mainly in driving neutral state. When the traffic state is congested or severely congested (the average value of congestion is 2.212), drivers are mainly in high anger state. In a word, as the congestion level grows, the anger intensity generated by drivers also increases. The conclusion can provide helpful references for traffic control or guidance concerned by traffic management authorities. From Tab. 6, we can further learn that the elasticity values $\left(E_{10}\right)$ of traffic congestion for all driving states are smaller than 1, which means traffic congestion is not a decisive factor for inducing driving anger.

\section{(4) Red light waiting time}

Except for traffic jams, long or frequent red lights are also an important factor for drivers' anger in the fast-paced urban life nowadays. As can be seen from Tab. 6, when the average value of red light waiting is 0.876 , namely, the waiting time ranges between short and comparably short, drivers are mainly in driving neutral state. When the red light waiting time ranges between comparably long and long (the average value is 2.038 ), drivers are mainly in high anger state. In a word, with the increase of red light time, the anger intensity generated by drivers also grows, leading to more dangerous behavior like rapid acceleration or hard brake. The conclusion can provide some reference for the design of phase period of traffic signal lamp. From Tab. 6, we can further learn that the elasticity values $\left(E_{11}\right)$ of red light time for all driving states are less than 1 , which means red light time is not a decisive factor for inducing driving anger. Moreover, the elastic value of red light time for high anger was 0.914 , significantly higher than that of other three driving states, which indicates that the influence of red light time change on inducing probability of high anger state was significantly greater than other driving states.

\section{(5) Pressure of time}

Based on the definition of "road rage", a driver is easily to be involved in angry driving or aggressive driving when he or she encounters an incentive situation under the pressure of life and work. As illustrated in Tab. 6, with the increase of anger intensity, the average value of time pressure also soars, which means a driver will become angrier when meeting the incentive situations under more time pressure, possibly leading to more aggressive behavior finally. This conclusion can provide some ideas for the design of human-machine interaction interface in connected intelligent vehicle. Namely, when the driver's high pressure is detected, some soft and relaxing music can be released to relieve the pressure of the driver. From Tab. 6 , we can further learn that the elasticity values $\left(E_{12}\right)$ of time pressure for all driving states are less than 1 , which means time pressure is not a decisive factor for inducing driving anger. Moreover, the elastic value of red light time for high anger was $0.965,1.2 \sim 2.3$ times that of other three driving states, which indicates under the medium or high time pressure (average value of 2.545), the probability of high anger outburst is 1.2 2.3 times that of the other three driving states.

\section{(6) Illegal traffic behaviors from surrounding people}

As everyone knows, driving process is essentially a process of human-vehicle-road-environment interaction. The driving environment, also called driving situation, refers to general traffic environment like congestion and traffic behaviors from surrounding people like jaywalk or cutting in line. Based on the self-reports from the subjects recruited in this study, we can find that the incentive 
driving situations are easily to induce drivers' anger while driving. As indicated in Tab. 6, with the increase of the seriousness of illegal traffic behaviors from surrounding traffic participants, the drivers' anger intensity also increases. The conclusion can provide reference for driver license training department and traffic management and law enforcement department to improve traffic quality of all traffic participants, such as civilized driving and travel. The elasticity values $\left(E_{13}\right)$ of illegal behaviors from surrounding people for all driving states are all greater than 1 , indicating that this influencing factor is rich in elasticity to the occurrence of driving anger, that is, illegal behaviors from surrounding people is a decisive factor for inducing driving anger. Particularly, the elasticity value of illegal behaviors from surrounding people for high anger was two times that of neutral state, indicating that the possibility of high anger induced was two times that of neutral states when the driver encountered illegal traffic behaviors around with average value of 2.785 .

\subsection{Precision Analysis of the Proposed Model}

In order to further verify the validity of the model proposed in this paper, it is necessary to analyze the precision of the model. Subject 9\# was arbitrarily selected as the test object, and the data for related personal attributes and incentive situations are listed as follow: $X_{1}=1, X_{2}=$ 29, $X_{3}=2, X_{4}=6, X_{5}=3, X_{6}=0, X_{7}=1, X_{8}=1, X_{9}=1, X_{10}$ $=2, X_{11}=2, X_{12}=1, X_{13}=3$. According to Eqs. (8) to (11), the value of unity function for the four driving anger states can be computed, then, the selected possibility for the four driving anger states can be calculated, shown as Tab. 7.

Table 7 Utility function value and presentation probability of driving states of subject 9\#

\begin{tabular}{|c|c|c|c|c|}
\hline Driving anger states & $A$ (Neutral) & $B$ (Low anger) & $C$ (Medium anger) & $D$ (High anger) \\
\hline Utility function value & 10.0283 & 13.9905 & 14.3894 & 16.118 \\
\hline Presentation probability & $0.17 \%$ & $9.17 \%$ & $13.67 \%$ & $76.99 \%$ \\
\hline
\end{tabular}

As can be seen from Tab. 7, subject 9\# had the highest probability $(76.99 \%)$ of presenting high anger states, correspondingly, it is determined that subject 9\# would present a high anger state. Based on the personal attributes values and incentive situations values, we know subject 9\# is a young male with short driving years; he is a nonprofessional driver with choleric temperament and has never been involved in a traffic accident. During the experiment, he was finally induced with high anger when he happened to encounter a surrounding car's cutting in line in a congested traffic. The result further verifies that a driver with choleric temperament is inclined to be emotional and aggressive.

A total of 825 driving samples in the naturalistic experiments were randomly selected, including 367 neutral samples, 217 low anger samples, 160 medium anger samples and 81 high anger samples, to test the proposed model by comparing real driving states from the subjects' self-reports with the results predicted by the proposed model. The precision indexes such as true positive rate $(T P R)$, false positive rate $(F P R)$ and accuracy $(A c c)$ of the proposed model are obtained. The calculation formula is as follows:

$$
\begin{aligned}
& T P R=\frac{T P}{T P+F N} \\
& F P R==\frac{F P}{T N+F P} \\
& A c c=\frac{T P+T N}{T P+F N+T N+F P}
\end{aligned}
$$

where, TP is the number of positive samples correctly classified; $F P$ is the number of negative samples which are falsely classified into positive ones; $T N$ is the number of negative samples correctly classified; $F N$ is the number of positive samples which are falsely classified into negative ones. Here, driving anger samples with certain intensity is supposed to be positive while the other driving anger samples are supposed to be negative. The calculation

\begin{tabular}{|c|c|c|c|c|c|c|c|c|}
\hline & $\begin{array}{l}\text { Classified } \\
\text { as neutral }\end{array}$ & $\begin{array}{c}\text { Classified as } \\
\text { low anger }\end{array}$ & $\begin{array}{l}\text { Classified as } \\
\text { Medium anger }\end{array}$ & $\begin{array}{c}\text { Classified as } \\
\text { high anger }\end{array}$ & Sum & $T P R$ & $F P R$ & $A c c$ \\
\hline Neutral & 291 & 42 & 28 & 6 & 367 & $79.29 \%$ & $5.46 \%$ & \multirow{4}{*}{$78.30 \%$} \\
\hline Low anger & 16 & 166 & 25 & 10 & 217 & $76.50 \%$ & $9.87 \%$ & \\
\hline Medium anger & 7 & 14 & 123 & 16 & 160 & $76.88 \%$ & $9.32 \%$ & \\
\hline High anger & 2 & 4 & 9 & 66 & 81 & $81.48 \%$ & $4.30 \%$ & \\
\hline
\end{tabular}
results are shown in Tab. 8.

Table 8 Precision of prediction model of driving anger states based on MNL

As can be seen from Tab. 8, the TPR of high anger samples is the highest, reaching $81.48 \%$, followed by neutral samples, and TPR of low anger samples is the lowest, yet above $75 \%$. The lowest FPR is found in neutral samples, which is only $5.46 \%$, followed by high anger samples. The higher FPR is found in low and medium anger samples, both of which are about $9 \%$. The overall accuracy of the four types of driving samples was $78.30 \%$, which indicates that proportion of driving samples correctly predicted by the proposed model is in good agreement with the actual self-reports, and the prediction of high anger samples is comparably more precise.
Therefore, this model is suitable to be used to predict driving anger states in real traffic environment.

\section{CONCLUSIONS}

The main goal of this paper is to figure out quantitative relationship between driving anger state and its influencing factors, as well as the impacting degree of the influencing factors on driving anger states with different intensity for taking the corresponding intervening measures. For instance, the traffic management authorities should put more emphasis on monitoring or warning for the decisive 
incentive situations mainly consisting of illegal traffic behaviors like cutting in line. On the other hand, driving license training departments should strengthen the psychological intervention for professional drivers with choleric or sanguineous temperament who are inclined to be aggressive during driving. Besides, with the rapid development of intelligent connected vehicle nowadays, the targeted intervening measures for driving states with different anger intensity which can be predicted by the proposed model may be applied in those intelligent vehicles. For example, when a driver is predicted with low anger state by advanced driving assistant system installed in the intelligent vehicle, soft intervening like relaxed music or warning can be released in advance by the assistant system. And hard intervening like brake/acceleration pedal or steering wheel control can be executed in advance by the assistant system when the driver is predicted with medium or high anger state.

Firstly, a special busy route was designed for the naturalistic experiments during which incentive situations like jaywalking, cutting in line, traffic congestion and waiting for red lights frequently occurred, especially in morning or evening rush hours. The subjects' self-reports verify that the novel induction method of driving anger proposed in this study is viable by stimulation of incentive situations in real traffic environment and extra paid if finishing the experiment ahead of reference time. Interestingly, Deffenbacher et al.[31] discovered that driving anger was mostly triggered in six common scenarios in U.S. including hostile gestures, discourtesy, slow driving, traffic obstructions, police presence and illegal driving. However, the survey reports obtained in this study show that driving anger triggering scenarios are distinctive in China, due to the differences in traffic rules, culture background, pace of life, safety awareness. Therefore, the results can provide theoretical support for key monitoring or targeted intervention of the influencing factors of driving anger for traffic management authorities and driving license training departments.

Secondly, after collecting personal attributes data by the related questionnaires and incentive situations data by coding of video replayed, and driving anger states data by the subjects' self-reports, a selection (prediction) model of driving anger states based on MNL was proposed in this study. The t-test result and McFadden determination coefficient $(0.367)$ shows the proposed model is suitable and reliable to predict driving anger using the personal attributes and incentive situations. Moreover, the accuracy of the proposed model even reaches 78.30\%. Most importantly, sensitivity analysis of the influencing factors to driving anger was conducted according to elasticity theory. The results show that the elasticity value of age, temperament and illegal traffic behaviors from surrounding people are all greater than 1 for neutral, low anger, medium anger and high anger driving state, which means the three influencing factors are decisive for inducing driving anger. Of course, other influencing factors do have impact on driving anger, but without decisive effect. Therefore, the proposed model could clarify the trigger mechanism of driving anger by quantifying the couple effect of those two kinds of influencing factors on driving anger.

Nevertheless, there still exist some limitations in this study. Firstly, as more male drivers were enrolled for statistical power, the number of females should be increased to improve universality of the proposed method in future. However, female drivers usually become angrier than males when encountering traffic block, and if they become angry, they are apt to present more adaptive adjustments and less aggression [10]. Then, a specific driving situation including more crowded routes, more red lights, more jaywalking, more slow cars ahead can be adopted to trigger more anger for female drivers. Besides physical movements and verbal aggressions, other measurements like physiological or facial expression characteristics can be used to show females' driving anger expression differences. Secondly, the naturalistic experiments proposed in this study were only carried out in Wuhan, a typical central metropolis in China. The succeeding experiments should be carried out in other typical cities like Chengdu, Guangzhou and Beijing, considering the differences of anger induction factors because of pace of life, safety awareness, traffic customs. Thirdly, in order to further improve the accuracy of the proposed model and explore more sensitive influencing factors, more personal attributes such as marital status, salary status, and fertility circumstance should also be taken into consideration. Fourthly, considering the relatively small number of participants, more discretization groups should be added for every variable to improve the accuracy of the proposed model. Fifthly, a lot more subjects should be recruited to comprehensively and deeply study the impact of personal attributes on driving anger in the future. For instance, personal disposition like personality, including agreeableness, extroversion, openness, responsibility, and emotional stability, should be well considered due to its serious impact on driving anger.

\section{Acknowledgement}

The present study is a supported project funded by Young Doctor Fund of Social Science program of Jiang XiProvince (17BJ42), Fund of Science andTechnology program of Education Department of Jiang Xi Province (GJJ180359) and Jiangxi University Humanities and Social Science Research Project (GL19204). National Nature Science Foundation of China (52062014, 62003220), Natural Science Foundation of Jiangxi Province (20202BABL212009).

\section{REFERENCES}

[1] Wu, C. Z. \& Lei, H. (2010). Review on the study of motorists' driving anger. China Safety Science Journal, 20(7), 3-8.

[2] NHTSA (2011). Traffic safety facts: a compilation of motor vehicle crash data from the fatality analysis reporting system and the general estimates system. U.S.A. Department of Transportation.

[3] Lei, H. (2011). The characteristics of angry driving behaviors and its effects on traffic safety. Master's thesis, Wuhan University of Technology, Wuhan, China.

[4] Dahlen, E. R., Martin, R. C., Ragan, K., \& Kuhlman, M. (2005). Driving anger, sensation seeking, impulsiveness, and boredom proneness in the prediction of unsafe driving. Accident Analysis and Prevention, 37(2), 341-348. https://doi.org/10.1016/j.aap.2004.10.006

[5] Sheila S., Alanna, M., Mohammad, E., Mohammad, K., \& Karen, W.(2000). Aggressive driving and road rage behaviors on freeways in sand ego, California: spatial and temporal analyses of observed and reported variations. Transportation Research Record, 1724, 7-13. 
https://doi.org/10.3141/1724-02

[6] Lajunen, T. \& Parker, D. (2001). Are aggressive people aggressive drivers? A study of the relationship between selfreported general aggressiveness, driver anger and aggressive driving. Accident Analysis and Prevention, 33(2), 243-255. https://doi.org/10.1016/S0001-4575(00)00039-7

[7] NHTSA (2007). Traffic safety facts 2007: a compilation of motor vehicle crash data from the fatality analysis reporting system and the general estimates system. Washington DC: National Highway Traffic Safety Administration.

[8] Lv, S. Y. (2016). The Ministry of Public Security will crack down on "road rage" violations such as forced lane changes and overtaking.

Retrieved from http://finance.chinanews.com/auto/2015/051/7266660.shtml

[9] Deffenbacher, J., Stephens, A., \& Sullman, M. (2016). Driving anger as a psychological construct: twenty years of research using driving anger scale. Transportation Research Part F: Traffic Psychology and Behavior, 42, 236-247. https://doi.org/10.1016/j.trf.2015.10.021

[10] González-Iglesias, B., Gómez-Fraguela, J. A., \& LuengoMartín, M. (2012). Driving anger and traffic violations: Gender differences. Transportation Research Part F: Traffic Psychology and Behavior, 15, 404-412. https://doi.org/10.1016/j.trf.2012.03.002

[11] Stephens, A. N. \& Groeger, J. A. (2011). Anger-congruent behavior transfers across driving situations. Cognition \& Emotion, 25(8), 1423-1438. https://doi.org/10.1080/02699931.2010.551184

[12] Putranto, L. S., Suryana, D. C., \& Bagakara, S. (2018). Development of Indonesian driving anger. $11^{\text {th }}$ Asia Pacific Transportation and the Environment Conference (APTE 2018), Advances in Engineering Research, (186), 164-169. https://doi.org/10.2991/apte-18.2019.30

[13] Feng, Z., Zhan, J., Ma, C., Lei, Y., Liu, J., Zhang W., \& Wang, K. (2018). Is cognitive intervention or forgiveness intervention more effective for the reduction of driving anger in Chinese bus drivers? Transportation Research Part F: Traffic Psychology and Behavior, 55, 101-113. https://doi.org/10.1016/j.trf.2018.02.039

[14] Smart, R. G., Stoduto, G., Mann, R. E., \& Adlaf, E. M. (2004). Road rage experience and behavior: vehicle, exposure, and driver factors. Traffic Injury Prevention, 5(4), 343-348. https://doi.org/10.1080/15389580490509482

[15] Shinar, D. (1998). Aggressive driving: the contribution of the drivers and the situation. Transportation Research Part F: Traffic Psychology and behavior, 1, 137-160. https://doi.org/10.1016/S1369-8478(99)00002-9

[16] Danaf, M., Abou-Zeid, M., \& Kaysi, I. (2015).Modeling anger and aggressive driving behavior in a dynamic choicelatent variable model. Accident Analysis and Prevention, 75, 105-118. https://doi.org/10.1016/j.aap.2014.11.012

[17] Sullman, M. J., Stephens, A., \& Yong, M. (2014). Driving anger in Malaysia. Accident Analysis and Prevention, 10(7), 1-9. https://doi.org/10.1016/j.aap.2014.04.019

[18] Li, F., Yao, X., Jiang, L., \& Li, Y. (2014). Driving anger in China: Psychometric properties of the driving anger scale (DAS) and its relationship with aggressive driving. Personality and Individual Differences, 68(10), 130-135. https://doi.org/10.1016/j.paid.2014.04.018

[19] Feng, Z., Lei, Y., Liu, H., Kumfer, W., Zhang, W., Wang, K., \& Lu, S. (2016). Driving anger in China: A case study on professional drivers. Transportation Research Part F: Traffic Psychology and Behavior, 42, 255-266. https://doi.org/10.1016/j.trf.2016.09.023

[20] Jovanovic, D., Lipovac, K., Stanojevic, P., \& Stanojevic, D. (2011). The effects of personality traits on driving-related anger and aggressive behavior in traffic among Serbian drivers. Transportation Research Part F: Traffic Psychology and Behavior, 14, 43-53. https://doi.org/10.1016/j.trf.2010.09.005

[21] Roidl, E., Siebert, F. W., Oehl, M., \& Höger, R. (2013). Introducing a multivariate model for predicting driving performance: The role of driving anger and personal characteristics. Journal of Safety Research, 47, 47-56. https://doi.org/10.1016/j.jsr.2013.08.002

[22] Shinar, D. \& Compton, R. P. (2004). Aggressive driving: an observational study of driver, vehicle, and situational variables. Accident Analysis and Prevention, 36(3), 429437. https://doi.org/10.1016/S0001-4575(03)00037-X

[23] Zhao, G. X. \& Zhao, J. F. (2000). Mental health handbook for adults. Science and Technology Press, Zhengzhou, China.

[24] Malyshkina, N. \& Mannering, F. (2009). Markov switching multinomial logit model: An application to accident- injury severities. Accident Analysis and Prevention, 41(1), 829838. https://doi.org/10.1016/j.aap.2009.04.006

[25] Feng, Z. X., Yuan, H. Z., Liu, J., Zhang, W. H., \& Liu, H. C. (2012). Influence of driver personal characteristics on vehicle velocity. Journal of Traffic and Transportation Engineering, 12(6), 89-96.

[26] Wan, P., Wu, C. Z., Lin, Y. Z., \& Ma, X. F. (2017). An onroad experimental study on driving anger identification model based on physiological features by roc curve analysis. IET Intelligent Transport Systems, 11(5), 290-298. https://doi.org/10.1049/iet-its.2016.0127

[27] Guan, H. Z. (2004). Disaggregate model --- a tool of traffic behavior analysis. China communication press, Beijing, China.

[28] Herrero-Fernández, D. (2011). Psychometric adaptation of the driving anger expression inventory in a Spanish sample: differences by age and gender. Transportation Research Part F: Traffic Psychology and Behavior, 14, 324-329. https://doi.org/10.1016/j.trf.2011.03.001

[29] Vujanic, M., Antic, B., Pesic, D., \& Savicevic, M. (2016). Testing the psychophysical characteristics of professional drivers - Can we identify unsafe drivers? Transportation Research Part F: Traffic Psychology and Behavior, 42, 104116. https://doi.org/10.1016/j.trf.2016.07.003

[30] Traffic Management Bureau of the Ministry of Public Security (2019). Road traffic accident statistical annual report of the people's Republic of China. Research Institute of Traffic Management, Ministry of Public Security, Wuxi, China.

[31] Deffenbacher, J. L., Oetting, E. R., \& Lynch, R. S. (1994). Development of a driving anger scale. Psychological Reports, 74(1), 83-91. https://doi.org/10.2466/pr0.1994.74.1.83

\section{Contact information:}

\section{Ping WAN}

School of Transportation and Logistics,

East China Jiaotong University, Nanchang, 330013, China

E-mail: pingw04@163.com

\section{Lixin YAN}

School of Transportation and Logistics,

East China Jiaotong University, Nanchang, 330013, China

E-mail: 879815845@qq.com

\section{Shan LU}

(Corresponding author)

Institute of Intelligence Science and Engineering,

Shenzhen Polytechnic, Shenzhen, 518055, China

E-mail: shanluszpt@163.com 